\title{
La cultura material de los tesoreros generales de la Santa Cruzada en el Burgos del siglo XVIII
}

Francisco Sanz de la Higuera

I.E.S. Torreblanca, Sevilla

CES.XVIII, núm. 25 (2015), págs. 317-336. 


\section{RESUMEN}

La documentación recopilada en los protocolos notariales, en especial los inventarios post-mortem de varios tesoreros generales de la Santa Cruzada de Burgos en la segunda mitad del Seiscientos y gran parte del Setecientos, permite un análisis crítico de sus niveles de fortuna y de los principales sesgos de su cultura material y una aproximación a la cultura de las apariencias y a las redes de influencia en las que se debatían de forma cotidiana o de una manera más extraordinaria. Sus interiores domésticos en vida y las mentalidades que se adivinan a través de sus últimas voluntades, posibilitan el descubrimiento de las estrategias que hubieron de adoptar ellos, sus hogares y los entramados en que estaban implicados.

La tesorería general de la Santa Cruzada no estaba al alcance de cualquiera. Disponían de significativos recursos económicos y financieros, una adecuada formación contable y nutridos contactos, cercanos o lejanos, en las intrincadas redes de la Administración. Eran testaferros de individuos muy poderosos, de La Corte, ubicados al final de una maquinaria aviesa y complicada, preñada de peldaños de poder, entre las cumbres más aristocráticas y los niveles intermedios, con los consumidores de las bulas en el extremo más débil y desprotegido. Abstenerse de ayunar tenía sus sobrecargas para unos, la mayoría, y sus beneficios para otros, una minoría afortunada y económicamente solvente.

Palabras clave

Santa Cruzada, Burgos, Siglo XVII y XVIII, cultura material, redes de influencia.

\section{Abstract}

The compiled information in the notarial registry, in special the probate inventories of several major treasurers of the Santa Cruzada in Burgos in the second half of Seventeen Century and great part of Eigthteen Century, allow a critical analisys of their levels of fortune an the main slant of the material culture and an aproximation to the appearance culture and to the influence net in which they were debated of daily form or in a more extarordinary way. Their domestic interiors in life and the mentalities that are guessed through their last wills, allow the discovery of the taken strategies, their homes and the studdings in which they were involved.

The general treasury of the Santa Cruzada was not scope of anyone. They had significant economic and financiers resources, a suitable accountig education and full contacts, nearby or distant, in the dense nets of the Administration. They were testaferros of very powerfull people, of the Cort, located at the final part of a difficult and complicated machine, full of steps of power, between the most aristocratic summit and the interval levels, with the consumers of the bulas in the most weak and disprotected extreme. To be abstain from fasting had their overload for ones, the majority, and its benefits for the others, a lucky and economicaly solvent minority.

Key Words

Santa Cruzada, Burgos, XVIIth and XVIIIth Centuries, material culture, influence nets. Recibido: 11 de enero de 2015. Aceptado: 15 de mayo de 2015. 


\section{Planteamientos generales}

Estas páginas no pretenden desentrañar la creación, el devenir y las peculiaridades más intrincadas de la Administración de la bula de la Santa Cruzada — sobre dichas problemáticas existen múltiples aproximaciones de gran calado e interés 1 —. Sencillamente se adentran en la consideración de la cultura material $^{2}$ de quienes fueron tesoreros generales de dicha bula en la ciudad de Bur-

1 Es imprescindible citar, como uno de los grandes clásicos, aunque ya bastante obsoleto y claramente descompensado hacia el período medieval, la obra de José GoÑ GazTambide, Historia de la bula de la Santa Cruzada, Vitoria, Editorial del Seminario, 1958. Más progresistas y modernistas, las propuestas de análisis de Alberto Marcos Martín, «Tráfico de indulgencias, guerra contra infieles y finanzas regias. La bula de Cruzada durante la primera mitad del siglo XVII», en Miguel Rodríguez Cancho (coord.), Historia y perspectivas de investigación. Estudios en memoria del profesor Ángel Rodríguez Sánchez, Badajoz, Editora Regional de Extremadura, 2002, págs. 227-236. Véanse también, entre otras y sin ánimo exhaustivo, las propuestas de Modesto UlloA, La Hacienda Real de Castilla en el reinado de Felipe II, Madrid, Fundación Universitaria Española, 1977. David Alonso García, El erario del Reino. Fiscalidad en Castilla a principios de la Edad Moderna, 1504-1525, Valladolid, Junta de Castilla y León, 2007. Alonso Pérez De Lara, Compendio de las Tres Gracias de la Santa Cruzada, Subsidio y Excusado, Madrid, Imprenta Real, 1610. José Fernández Llamazares, Historia de la bula de la Santa Cruzada, Madrid, Eusebia Aguado, 1859. Ramón Hernández, La confesión y las indulgencias. Prerreforma y tradición, Salamanca, Editorial San Esteban, 1978. Lucía Carpintero Aguado, «Iglesia y Corte castellana en el siglo XVI. Contribución y tributos», Hispania Sacra, 84 (1989), págs. 547-567. István Szasdi León-Borja, «La introducción de la Bula de la Santa Cruzada en el Nuevo Mundo (1509-1525)» Cuadernos de Historia del Derecho y de las Ideas Políticas, 10 (2000), págs. 113-149. José Antonio Benito Rodríguez, «Organización y funcionamiento de los tribunales de Cruzada en Indias», Revista de Estudios Histórico-Jurídicos, 22 (2000), págs. 169-190 y Eugenio Serrano Rodríguez y Miguel Fernando Gómez VozMEDIANo, «Imprenta, dinero y fe: la impresión de bulas en el convento dominico de San Pedro Mártir de Toledo (1483-1600)», Tiempos Modernos, 27 (2013), págs. 1-65.

2 Son muchas, y de excelente calidad, las propuestas sobre cultura material. Descuellan, no obstante, los análisis de Máximo García Fernández, «La cultura material doméstica en la Castilla del Antiguo Régimen», en Máximo García Fernández y M.a Ángeles Sobaler Seco (coords.), Estudios en homenaje al profesor Teófanes Egido, Valladolid, Junta de Castilla y León, 2004, Vol. II, págs. 249-270. Isabel dos Guimaraes SÁ y Máximo García Fernández (dirs.), Portas adentro: comer, vestir, habitar (ss. XVI-XIX), Coimbra y Valladolid, Universidad de Coimbra y Universidad de Valladolid, 2010. Fernando Ramos Palencia, Pautas de consumo y mercado en Castilla, 1750-1850. Economía familiar en Palencia al final del Antiguo Régimen, Madrid, Sílex, 2010. Jesús Aguado de los Reyes, Riqueza y sociedad en la Sevilla del siglo XVII, Sevilla, Universidad de Sevilla, 1994. Weatherill, Lorna, Consumer behaviour and Material Culture in Britain, 1669-1760, Londres y Nueva York, Routledge, 1996. Carole Shammas, The Preindustrial Consumer in England and America, Oxford, Clarendon Press, 1990. Micheline Baulant, Anton Schuurman y Paul Servais (eds.), Inventaires après-décès et ventes de meubles: Apports à une histoire de la vie économique et quotidienne (XIV-XIXe siècles), Louveain-laNeuve, Academia, 1987. Ad Van Der Woude y Anton Schuurman (eds.), Probate inventoires. A new source 
gos a lo largo de la segunda mitad del siglo XVII y gran parte del siglo XVIII. Por supuesto, tales asertos nos impelen a zambullirnos, en la medida de lo posible, en las culturas de las apariencias ${ }^{3}$ en que se encontraban implicados y las redes de influencias ${ }^{4}$ a las que tenían acceso y a las que se encontraban supeditados. Llegar a la tesorería general de la bula de la Santa Cruzada constituía una situación coyuntural derivada del manejo de estrategias personales, familiares, estamentales y de «clase» que penetraban, muy profundamente, en la idiosincrasia de sus titulares y de quienes les avalaban, promovían, alentaban y sostenían, responsables de la Administración del Estado tanto en tiempos de los últimos Austrias como de los primeros Borbones.

Para la reconstrucción de la cultura material, las mentalidades y las redes de influencia de dichos individuos, y hogares, contamos con un yacimiento documental insustituible, los protocolos notariales, y dentro de ellos, en especial, los inventarios post-mortem ${ }^{5}$ y/o los inventarios de bienes por nupcias y las últimas voluntades ${ }^{6}$. Además, y de manera complementaria, al menos para lo

for the historical study of wealth, material culture and agricultural development, Utrech, Hes Publisher, 1980 y Françoise Waro-Desjardins, La vie quotidienne dans Le Vexin au XVIIT siècle dans l'intimité d'une société rurale, Pontoise, Editions du Vallhermeil, 1992.

3 Véanse, sin ánimo exhaustivo, Daniel Roche, La culture des apparences. Une histoire du vêtement, XVI ${ }^{e}$-XVIII siècles, París, Fayard, 1989. Antonio Álvarez-Ossorio Alvariño, "Lujo y movilidad social. Iglesia y Corona frente a la quiebra de la distinción en Castilla (siglos XVI-XVIII), en II Congreso italo-ibérico di Demografia Storica, Savona, ADES/SIDES, 1992, Vol. II, págs. 752-767. Jaime ValenzuELa MárQ̨uez, «Afán de prestigio y movilidad social: los espejos de la apariencia», en Cristian GazmurRi y Rafael SAGredo (eds.), Historia de la vida privada en Chile, Santiago de Chile, Taurus, 2005, págs. 71-93 y Francisco José SANZ DE La Higuera, «La vestimenta del clero en el Burgos del XvIII», Revista de Historia Moderna, 31 (2013), págs. 127-146.

4 Descuellan con luz propia las excelentes propuestas de José M. a Imízcoz BEunza, Élites, poder y red social. Las élites del País Vasco y Navarra en la Edad Moderna, Bilbao, Universidad del País Vasco, 1996 y Redes familiares y patronazgo. Aproximación al entramado social del País Vasco y Navarra en el Antiguo Régimen (siglos XV-XIX), Bilbao, Universidad del País Vasco, 2001. José M. a Imízcoz BeunZa y Oihane OLIveri Ковта (eds.), Economía doméstica y redes sociales en el Antiguo Régimen, Madrid, Sílex, 2010. Cosme Jesús Gómez Carrasco, «Parientes, amigos y patronos. Red, movilidad y reproducción social en la burguesía y la élite de poder a finales del Antiguo Régimen (Albacete, 1750-1808)», Stvdia Historia, Historia Moderna, 29 (2007), págs. 427-463 y Bartolomé Yun CasaliLla, Las redes del Imperio. Élites sociales en la articulación de la Monarquía Hispánica, 1492-1714, Madrid, Marcial Pons, 2009.

5 Máximo García Fernández, Herencia y patrimonio familiar en la Castilla del Antiguo Régimen (1650-1835). Efectos socioeconómicos de la muerte y la partición de bienes, Valladolid, Universidad de Valladolid, 1995. Los protocolos notariales han sido recopilados en el Archivo Histórico Provincial de Burgos -AHPB. PN en lo sucesivo-.

6 Máximo García Fernández, Los castellanos y la muerte. Religiosidad y comportamientos colectivos en el Antiguo Régimen, Valladolid, Junta de Castilla y León, 1996. Juan Postigo Vidal, «Los escenarios de la muerte. Cultura material, religiosidad y ritual en las postrimerías durante la Edad Moderna», en M. José Pérez Álvarez y Alfredo Martín García (eds.), Campo y campesinos en la España Moderna. Culturas políticas en el mundo hispano, León, Universidad de León, 2012, Vol. II, págs. 2047-2058 y Francisco SANZ DE LA HIGUERA, «La terrible f(r)actura de la muerte. Fallecer en el Burgos del Setecientos», Cuadernos de Investigación Histórica, 23 (2006), págs. 251-284 
tocante a don Pedro Tomé González, disponemos de un importante volumen de documentación procedente del quehacer notarial y, a la postre, la información extraída de su memorial en las Respuestas Particulares elaboradas para el Catastro del marqués de la Ensenada ${ }^{7}$. El trabajo de archivo, de «tercer nivel» ${ }^{8}$, ha posibilitado la recopilación de los niveles de fortuna de tres tesoreros generales de la Santa Cruzada ${ }^{9}$. En primera instancia, el inventario post-mortem ${ }^{10}$ de don Juan de Herrera $(1651)^{11}$. En segundo término, el de don Dionisio Garma de la Puente $(1702)^{12}$ y, a la postre, el del tendero en sus inicios y posteriormente asentista de la pólvora, mayordomo del Cabildo catedralicio ${ }^{13}$ y empresario lanero ${ }^{14}$ don Pedro Tomé González (1764) ${ }^{15}$.

La documentación histórica recopilada tiene en el acervo bibliográfico un imprescindible instrumento auxiliar. Nos debatimos, dialécticamente, en la estructura de una doble hélice genética de carácter historiográfico, entre lo que presuntamente sucedió en lo cercano, en la micro-historia de Burgos, y el con-

7 Archivo de la Diputación Provincial de Burgos. Catastro de Ensenada. Respuestas Particulares. Libros 344-349.

$8 \quad$ Véanse a este respecto las propuestas metodológicas de Chaunu, 1973, 105/125. David GonzÁlez Cruz, Religiosidad y ritual de la muerte en la Huelva del siglo de la Ilustración, Huelva, Universidad de Huelva, 1993, págs. 21-22 y Carlos Alberto González Sánchez, Los mundos del libro. Medios de difusión de la cultura occidental en Las Indias en los siglos XVI y XVII, Sevilla, Universidad de Sevilla, 2001, pág. 26.

9 Se me puede achacar, y con razón, que disponer de la documentación de únicamente tres hogares es una muy escasa muestra, que, por supuesto, no es representativa de ningún grupo social y que, en efecto, constituyen una modesta aproximación a un grupo de individuos que ejercieron en un cierto período de sus trayectorias vitales una actividad asentista y cobradora de rentas, circunstancias de las cuales difícilmente pueden sacarse conclusiones representativas. Ojalá dispusiera de muchos más administradores generales de la bula de la Santa Cruzada en el Burgos del Antiguo Régimen. Lanzo el reto a la comunidad de investigadores para que clonen este mi análisis en sus propias localidades y que alguien con mucha más sabiduría e inteligencia que yo efectúe un estudio de contraste de la representatividad de dichos tesoreros y su cultura material en el ámbito español de la Edad Moderna.

10 En lo sucesivo citado como IPM.

11 AHPB. PN. Juan Díez del Real. Legajo 6515 (13 de junio de 1651), folios 645-679.

12 AHPB. PN. Lázaro Santamaría. Legajo 6862 (19 de diciembre de 1702), folios 887-917.

13 Francisco Sanz de la Higuera, «Los mayordomos de la mesa capitular de la Catedral de Burgos en el Setecientos (1707-1782)», Trocadero, 25 (2013), págs. 117-146.

14 Véanse las propuestas de Francisco SAnz DE LA Higuera, «Las "restauraciones" del Consulado de Burgos en el siglo XvIII», Historia, Instituciones, Documentos, 29 (2002), págs. 429-458; «Pólvoras, municiones, lanas, granos, bulas y papel sellado (1704-1764): Don Pedro Tomé González y la hora navarra en el Burgos del siglo XVIII», Boletín de la Institución Fernán González (BIFG), 227 (2003), págs. 375-411; «Estando como estamos juntos y congregados en la casa de dha Contratazión como lo acostumbramos»: Luces y sombras en el Consulado de Burgos, BIFG, 229 (2004), págs. 377-414; "“Esquileo y tixera” en Burgos: La pila de lanas del Hospital del Rey. Siglos XVI, XVII y XVIII» (I), en BIFG, 233, (2006), págs. 315-340 y (II), BIFG, 234 (2007 a), págs. 245-268 y «Un esperpéntico asuntillo de Estado: La casa de los Tomé en el Setecientos burgalés», Cuadernos de Estudios del Siglo XVIII, 21 (2011), págs. 287-318.

15 AHPB. Justicia Municipal (JM). Juan Antonio Fernández Guilarte. Legajo 977 (14 de julio de 1765), folios 21-127. Para Tomé González disponemos, además, de otros dos IPM en AHPB. PN. José de Mata. Legajo 6917 (11 de enero de 1703), folios 218-229 y AHPB. PN. Martín de Robredo. Legajo 6964 (14 de enero de 1724), folios 340-353. 
traste diferencial con lo acontecido en otras latitudes durante el mismo período de tiempo. A mi entender, es de notable aprovechamiento, por su calidad y claridad, el trabajo del profesor Marcos Martín sobre la bula de Cruzada en el siglo XVII ${ }^{16}$ y de notoria calidad la propuesta de Valenzuela Márquez ${ }^{17}$. Excelentes son las sugerencias que ha propuesto Catalán Martínez ${ }^{18}$. No es menor el sesgo de Torres Gutiérrez $^{19}$ y Benito Rodríguez ${ }^{20}$ o los análisis de Serrano Rodríguez y Gómez Vozmediano $^{21}$. Resultan de notable ayuda las sugerencias de Bertrand y Dedieu ${ }^{22}$ y de Postigo Vidal ${ }^{23}$. Sobre los niveles de fortuna y las estrategias fúnebres de los administradores de la bula véanse los hallazgos de Rojo Vega ${ }^{24}$. No desmerecen en nada los trabajos de Sanz Ayán para las problemáticas de los asientos de suministro y los arriendos de rentas y de percepción de impuestos y contribuciones ${ }^{25}$.

\section{Niveles de fortuna de los tesoreros generales de la Santa Cruzada en el Burgos del XVII y XVIII}

El devenir de los niveles de fortuna de los tesoreros generales de la Santa Cruzada de la ciudad de Burgos y su Arzobispado — véase gráfico I- muestra

16 Alberto Marcos Martín, «Tráfico de indulgencias...», 2002, págs. 227-236.

17 Jaime Valenzuela Márǫuez, «Afán de prestigio...», 2005, págs. 71-93.

18 Elena Catalán MarTínez, El precio del purgatorio. Los ingresos del clero vasco en la Edad Moderna, Bilbao, Universidad del País Vasco, 2000, págs. 201-243.

19 Alejandro Torres GUTIÉRREz, «Implicaciones económicas del miedo...», 2000.

20 José Antonio Benito Rodríguez, «Organización y funcionamiento ...», Revista de Estudios Histórico-Jurídicos, 22, (2000), págs. 169-190.

21 Eugenio Serrano Rodríguez y Miguel Fernando Gómez Vozmediano, «Imprenta, dinero y fe ...», Tiempos Modernos, 27 (2013), págs. 1-65.

22 Michel Betrand, y Juan Pierre Dedieu, « ¿Vino joven en odres viejas? Prosopografía y relaciones sociales en la Monarquía hispánica», en Miguel Ángel Rodríguez Cancho (coord.), Historia y perspectivas de investigación ..., 2002, págs. 31-37. Véanse igualmente a este respecto Úrsula VonES-LIEBENSTEIN, «El método prosopográfico como punto de partida de la historiografía eclesiástica», Anuario de Historia de la Iglesia, 14 (2005), págs. 351-364 y Adeline Daumard, «Les génealogies sociales. Un des fondaments d'histoire sociale comparative et quantitative», Annales de démographie historique, 1 (1984), págs. 9-24.

23 Juan Postigo Vidal, «Los escenarios de la muerte ...», 2012, Vol. II, págs. 2047-2058.

24 Anastasio Rojo VEGA, «1554. Inventario de Álvaro de Benavente, administrador de la Bula», [En línea]. Consultado el 14 de marzo de 2014. URL: anastasiorojovega.com/attachment/article/393/ 1554\%20 ALVARO\%20de\%BENAVENTE.pdf y «1554. Testamento de Álvaro de Benavente», [En línea]. Consultado el 14 de marzo de 2014. URL: anastasiorojovega.com/indix.php?option=com_content \&vies=article\&id=644. testamento-y-codicillo-de-alvaro-de-benavente\&catid=6:siglo-de-oro\&temid=9.

25 Carmen SAnZ Ayán, «La figura de los arrendadores de rentas en la segunda mitad del siglo XVII», Hispania, 165 (1987), págs. 203-224. Véase también las excelentes propuestas de Rafael TorRes SÁnchEz, «Servir al rey», más una comisión. El fortalecimiento de los asentistas en la corona española durante la segunda mitad del siglo XVIII», en Pablo Fernández Albadalejo (ed.), Monarquía, Imperio y pueblos en la España Moderna, Alicante, Caja de Ahorros del Mediterráneo y Universidad de Alicante, 1997, págs. 149-167. 

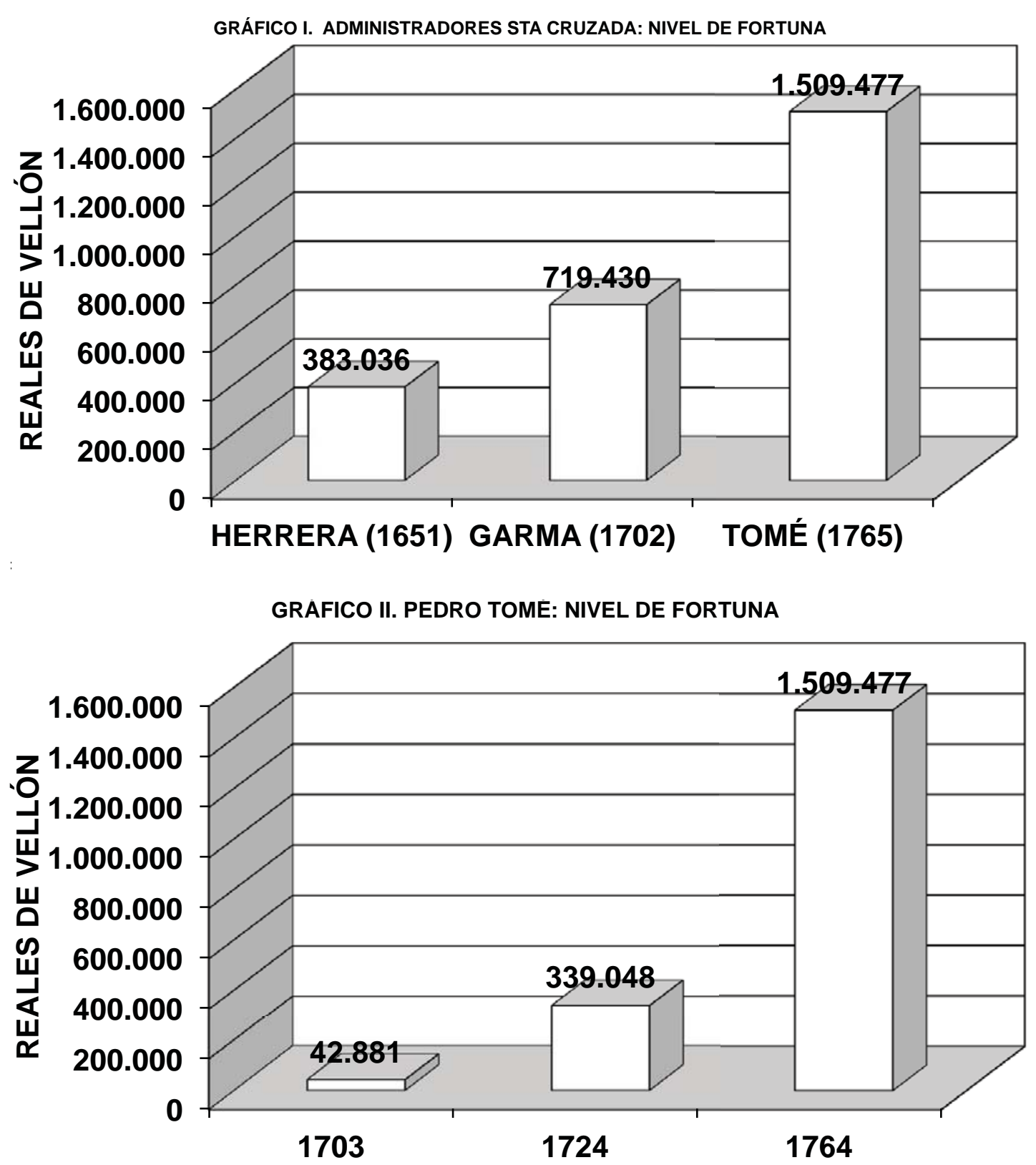

una progresión muy significativa, dado que, en la práctica, duplica su montante en cada uno de los IPM a que hemos tenido acceso. Fue ésta una tendencia generalizada en muchos de los «asientos» y obligaciones de suministro de la España del Antiguo Régimen ${ }^{26}$.

Tales incrementos adquieren, incluso, mucha más trascendencia si traemos a colación el sesgo diacrónico de los niveles de fortuna del citado don Pedro

26 Francisco Sanz de la Higuera, «Los mayordomos de la mesa capitular...», Trocadero, 25 (2013), págs. 117-146. 
Tomé — véase gráfico II—. En los momentos en que, por nupcias o por fallecimiento, le fueron efectuados los quehaceres notariales de inventario de bienes el tesorero general de la Santa Cruzada más eminente y popular del Setecientos disponía de un patrimonio libre ${ }^{27}$ creciente, hasta alcanzar unos niveles de renta y de fortuna sobresalientes y opíparos del Burgos del siglo xvIII. Indudablemente, fue en las pilas de lanas y en la distribución de las bulas de Cruzada donde se centra gran parte de su éxito profesional y socioeconómico ${ }^{28}$.

En la cotidianeidad del gigantesco entramado generado por la Corona española para el control de la bula de la Santa Cruzada, «los tesoreros que se hacían cargo por asiento de la distribución y la recaudación de la limosna eran personas de solvencia económica, generalmente mercaderes [ya sea en España o en el Nuevo Mundo], si bien encontramos gentes de otras profesiones, tales como profesores de la Universidad, militares, etc.» ${ }^{29}$. La apetencia por desempeñar dicho oficio estribaba, además de las exenciones que la Cruzada proporcionaba y el prestigio y familiar que suponía, en la obtención de un beneficio económico por su cometido, que oscilaba entre el 14 y el 20 por ciento de la recaudación, cantidad que lo hacía mucho más atractivo que la inversión en juros, en censos hipotecarios o en propiedades inmuebles para el arrendamiento.

Requisito esencial era que fueran «...buenas personas y de confianza y habilidades cuales convenga para la administración y cobranza de la dicha Santa Bula ${ }^{30}$. En los tres tesoreros generales de Burgos se daban, sin lugar a dudas, todas esas características. Las cantidades de dinero en efectivo que atesoraban en sus viviendas no les hacía, en el Burgos del XVII y XVIII, aparecer como grandes banqueros, que no lo fueron, pero sí como pequeños y medianos «capitalistas» autóctonos ${ }^{31}$, intermediarios de reconocido prestigio, experiencia en los asientos estatales y dotados de nutridas relaciones nacionales e internacionales que asumieron unos quehaceres de enorme trascendencia. La necesidad de los fieles católicos de «ganar indulgencias, lograr la remisión de los pecados o re-

27 Los bienes, menajes y pertrechos aparecidos en los inventarios post-mortem eran únicamente los bienes libres y no los de mayorazgo, que también los había, si bien no eran, salvo raras ocasiones contemplados o tasados.

28 Francisco Sanz de la Higuera, «"Esquileo y tixera”...»(II), BIFG, 234 (2007), págs. 245-268. Véase, desde una perspectiva más general, las magníficas obras de Ricardo Franch Benavent, Crecimiento comercial y enriquecimiento burgués en la Valencia del siglo XVIII, Valencia, Institució «Alfons el Magnanim», 1989 y El capital comercial valenciano en el siglo XVIII, Valencia, Universidad de Valencia, 1986. Véase también Manuel Moreno Alonso, Retrato de familia andaluza con Las Indias al fondo. El Memorial de El Pintado (1697-1780), Sevilla, Alfar, 2000.

29 José Antonio Benito RodríGuez, «Organización y funcionamiento...», Revista de Estudios HistóricoJurídicos (REHJ), 22 (2000), pág. 169.

30 José Antonio Benito Rodríguez, REHJ, 22 (2000), pág. 171.

31 Elena Catalán Martínez, El precio del purgatorio, 2000, pág. 235. 
ducir los días de ayuno y abstinencia» transformó una limosna en impuesto, y de ello sacaron provecho hombres — hogares- de negocios implicados en el «capitalismo nacional» $»^{32}$.

En la presunta ortodoxia de la bula de la Santa Cruzada, se fueron acumulando, con el paso del tiempo, diversas patologías funcionales. Las dos más sensibles derivaban, por una parte, de la «avidez y el desenfado de intrigantes indoctos y de mercaderes ávidos [que] fueron incubando prácticas viciosas» y, por otra, de la perversión popular que, según se recoge, por ejemplo, en las páginas del Lazarillo de Tormes, se producía cuando «hubo tanta prisa [en la toma de la bula] que casi ánima viviente en el lugar no quedó sin ella, como si fueran peras que se dieran en balde...sin predicar sermón ${ }^{33}$. Constituye un sarcasmo, con tintes brutales, que una limosna nacida para costear guerras circunstanciales a través del permiso, particular, para abstenerse de ayunar puntualmente se convirtiera en un gravamen de carácter fiscal con que la Corona financiaba cualquier problemática financiera — «Su exacción a lo largo de toda la Edad Moderna constituye un ingreso saneado de la Corona ${ }^{34}$ y predicada y consumida a destajo por la Iglesia y por la población, respectivamente. El miedo a la salvación del alma se había trocado en miedo a pasar hambre, con el correspondiente relajamiento en las conductas de los individuos, que a través de su adquisición compraban la liberación de las servidumbres restrictivas existentes para la ingesta de alimentos. Esta contaminación con prácticas e implicaciones profundamente materiales constituye un precoz aldabonazo secularizador y descristianizador, como otros muchos que fueron operando lentamente en la sociedad española. Ni tenía carácter patriótico ni era solamente espiritual, como en los siglos de las tinieblas ${ }^{35}$. En la época ilustrada era sencillamente un gravamen más, un impuesto sui generis, con «intereses creados» adaptados a los implicados, Estado, Iglesia, funcionarios, predicadores, tesoreros generales, consumidores...De todo ello se beneficiaban los tesoreros generales de la Santa Cruzada, dado que ninguno de ellos era paupérrimo y sus talegos no mermaban sino que crecían al ritmo de la avidez por consumo de la bula de vivos o de difuntos entre la población española del Antiguo Régimen ${ }^{36}$.

32 Alberto Marcos MarTín, «Tráfico de indulgencias...», 2002, pág. 228.

33 Ramón Carande, Carlos V y sus banqueros, Barcelona, Crítica, 1990, págs. 440-442. Citado en, y tomado de, Alejandro Torres GutiérRez, «Implicaciones económicas...», 2000, pág. 4.

34 Alejandro Torres Gutiérrez, «Implicaciones económicas...», 2000, pág. 4.

35 Véanse los análisis de Puigblanch, Antoni, La Inquisición sin máscara, Barcelona, Editorial Alta Fulla, 1988, pág. 487.

36 Es probable que la bula de Santa Cruzada no fuera una carga insufrible para nadie. La bula de vivos, la más popular, costaba 2 reales de plata — y desde 1624 en algunos lugares se autorizó la compra en vellón pagando $80 \mathrm{mrs}$ para compensar el premio — . Este coste no fue modificado desde el siglo Xvi hasta fi- 


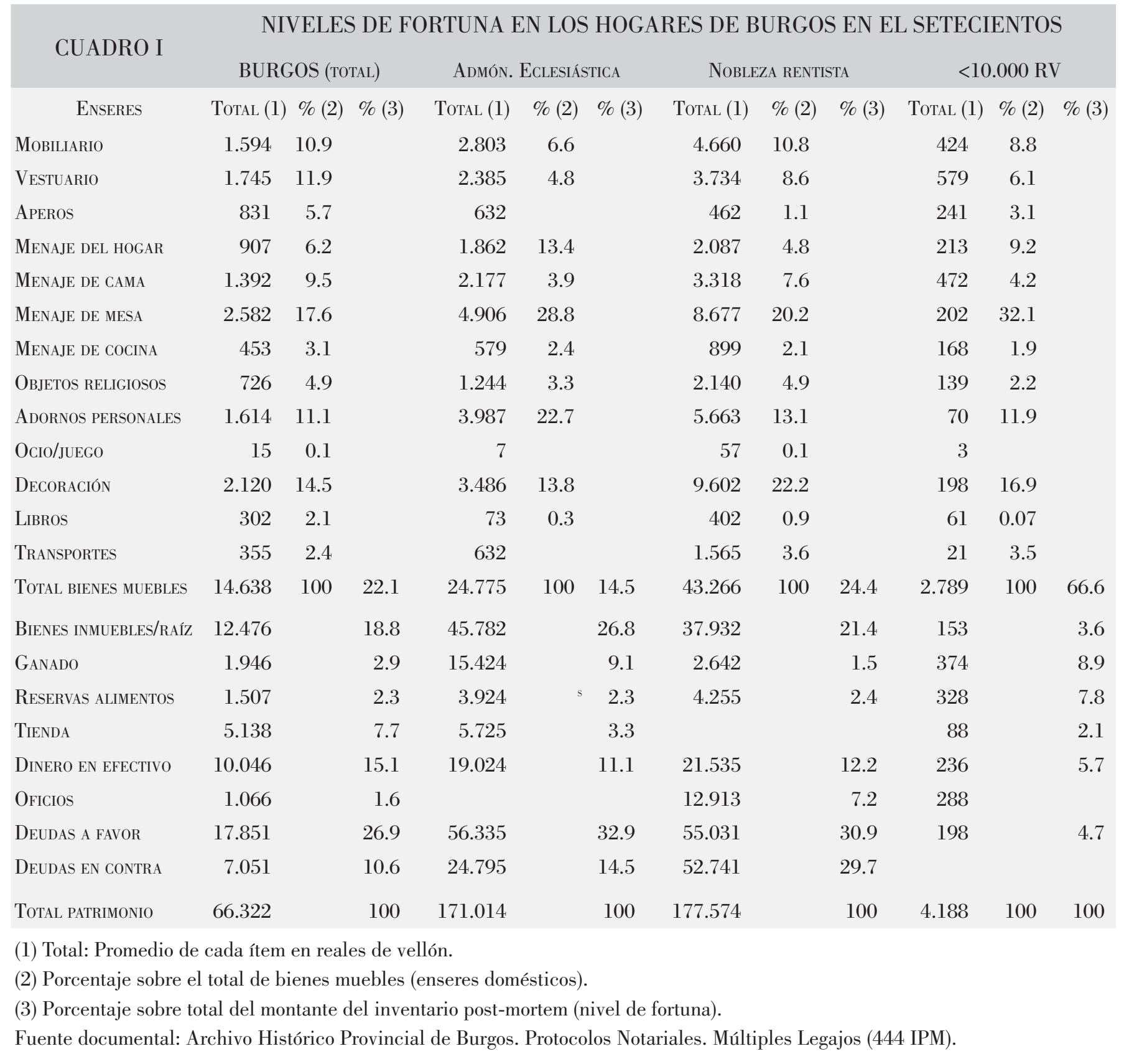

El volumen total de sus inventarios post-mortem contrasta nítidamente no sólo entre ellos mismos, con el citado incremento vertiginoso de sus niveles de fortuna a medida que se producían los relevos en la Administración General de la Santa Cruzada, sino especialmente con los promedios de la ciudad en

nales del XVIII. Los más paupérrimos no tenían que abonarla. Era un gasto anual que resultaba mínimo incluso para los poseedores de inventarios de bienes menos afortunados. 
su conjunto, los empleados de las Administraciones eclesiásticas ${ }^{37}$, los nobles $\operatorname{rentistas}^{38}$ y el patrimonio de aquellos que disponían de menos de 10.000 reales de vellón —véase cuadro I-.

Con el universo documental de 444 IPM para el Setecientos y de 37 para el siglo XVII, el Burgos del Seiscientos nos ofrece un promedio de 242.938 1/2 reales de vellón ${ }^{39}$ y el del siglo XVIII arroja un promedio de, aproximadamente, 66.000 reales, el de los empleados de las Administraciones de la Iglesia se eleva a poco más de 171.000 reales, casi lo mismo que el nivel de fortuna de los aristócratas rentistas, dirigentes del Concejo y terratenientes de la ciudad, con 177.574 reales. Nada que ver ninguno de ellos con el promedio de los más paupérrimos, cuyo promedio se desploma hasta los 4.188 reales de vellón. Don Juan de Herrera, don Dionisio Garma de la Puente y don Pedro Tomé González se encontraban instalados entre los más pudientes de sus respectivas etapas históricas — mediados del XVIII y principios y mediados del XVIII, respectivamenteLos 383.036 reales de vellón de Herrera colocaban su patrimonio en el cuarto lugar de los hallados para mediados del Seiscientos. El promedio de los IPM recopilados lo sitúa en una posición cómoda y aún privilegiada. Los 719.430 1² reales de Garma de la Puente lo ubican en el segundo puesto de los recopilados para 1700-1720 — cuyo promedio, 60.299 1² reales de vellón, acentúa más, si cabe, el rotundo patrimonio de don Dionisio-

Don Pedro Tomé fue uno de los hombres más acaudalados y respetados de gran parte del Setecientos. Su gradualmente creciente nivel de fortuna hizo de él, en especial merced a los negocios de las lanas, el segundo hogar más pudiente de la ciudad en el período 1740-1770 —el promedio que arrojan los IPM recopilados, 68.530 1/2 reales, atestigua también, como en el otros momentos, la valía y el devenir del patrimonio de Tomé González-. La tesorería general de la Santa Cruzada no era un asiento cualquiera y las redes de influencia en que se encontraban incardinados sus detentadores, aunque fuera temporalmente, les otorgaba una «línea caliente» con algunas de las más elevadas y cualificadas alcurnias del Reino. Los quehaceres de la Administración General de la Santa Cruzada en la ciudad en la que residían no les hizo ricos, ya lo eran

37 Se incluyen bajo tal denominación los notarios mayores de la Audiencia Arzobispal, los tesoreros del Hospital del Rey, los mayordomos y los contadores de la Mesa Capitular de la Catedral, los tesoreros generales, los notarios y los alguaciles de la Santa Cruzada y los administradores de Obras Pías.

38 Bajo dicho epígrafe contemplamos los hogares de extracción noble, de sangre o hidalgos, según el caso, algunos de ellos titulados, dedicados en esencia al disfrute de las rentas procedentes de sus mayorazgos y bienes libres y dirigentes, casi todos, del Concejo, como alcaldes y regidores perpetuos.

39 La escasa muestra recopilada para el siglo Xvir constituye a día de hoy, lamentablemente, un importante lastre en la calidad de los análisis, problemática que será rectificada, en la medida de lo posible, en próximas campañas de recogida de información en el AHPB. 
previamente, pero si que les catapultó hacia las esferas de poder cercanas a la aristocracia de La Corte, e impulsó, también, sus propios niveles de fortuna.

\section{Interiores domésticos y cultura de las apariencias}

Un análisis, crítico, pormenorizado de los bienes muebles e inmuebles libres —véase cuadro I, cuadro II y cuadro III- de los hogares y colectivos profesionales o socioeconómicos implicados en este estudio muestra cuáles eran los sesgos esenciales de sus comportamientos y estrategias en lo tocante al consumo y a sus capacidades adquisitivas. Un primer hecho significativo era que los tesoreros generales de la Santa Cruzada gustaban de disponer buenas mesas, preñadas de menajes de plata, cuberterías y mantelerías de notable calidad y cantidad, a lo que se añadirían excelentes menúes alimenticios ${ }^{40}$. En ello se comportaban a la usanza de sus «compadres» de las Administraciones eclesiásticas y de la nobleza rentista y dirigente del Concejo, o incluso con más fruición. En ambas categorías socioprofesionales, los menajes de mesa significaban alrededor del $20 \%$ de los bienes muebles. En los hogares más modestos, es decir, aquellos que no superaban los 10.000 reales de patrimonio, los menajes de mesa difícilmente superaban el $7 \%$. Don Juan de Herrera sobresalía abiertamente en este capítulo de la cultura material. Dichos menajes atesoraban el 40,3\% de sus bienes domésticos. Don Dionisio Garma acumulaba en tales menesteres el 28,8 \% de sus enseres del interior hogareño y don Pedro Tomé el $25.9 \%$. Era, con mucha diferencia, el más importante capítulo de inversión y consumo de sus establecimientos familiares.

Un segundo escalón en las estrategias desplegadas por los tesoreros generales de la Santa Cruzada en su búsqueda de preeminencia, elevada cultura material y confort y en demostrar unas apariencias de boato y magnificencia se materializa en unos interiores domésticos bien surtidos en lo tocante al vestir la casa con objetos y menajes de decoración de lujo. El más proclive a tal despliegue fue don Juan de Herrera, con un $24.8 \%$ de sus bienes muebles atesorados en dicho epígrafe, seguido por Garma $(13,8 \%$ y, en última instancia, por Tomé González (10,9\%). También en tales menesteres los tesoreros generales demostraban un talante más ornamentista y edulcorante de sus interiores domésticos

40 M. ${ }^{a}$ Ángeles PÉrez SAMPER, Mesas y cocinas en la España del siglo XVIII, Oviedo, Trea, 2011. Véase también Francisco SANZ DE LA Higuera, «Aproximación a la mesa de los burgaleses. Cuberterías y platos en el Setecientos», en Máximo García Fernández (Dir.), Cultura material y vida cotidiana Moderna: Escenarios, Madrid, Ś́lex, 2013, págs. 183-195 y «A la mesa de los burgaleses del Setecientos. Mantelerías, cuberterías, vidrios y vidriados», Investigaciones Históricas, 34 (2014), págs. 107-130. 


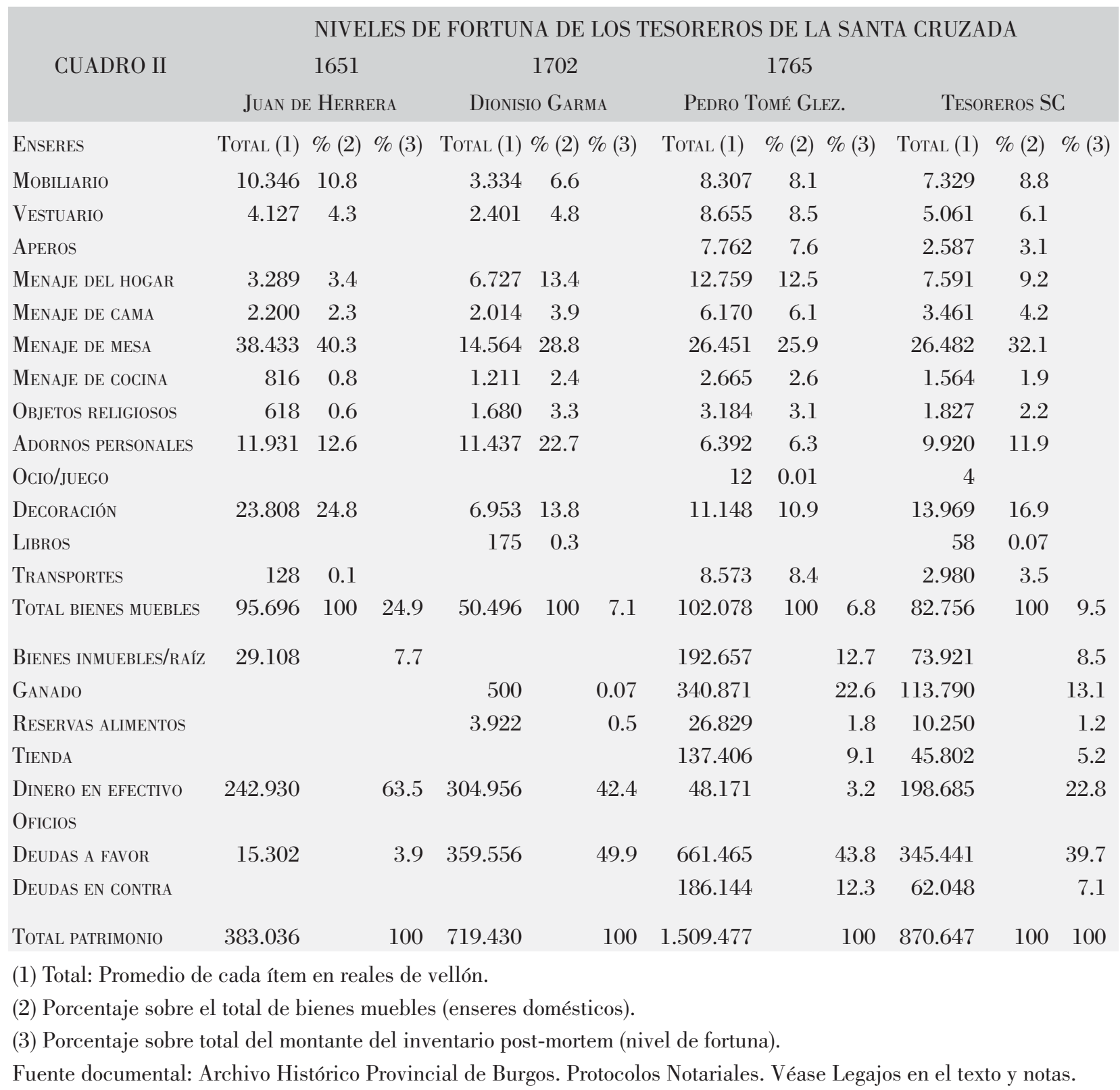

que los hogares de la ciudad en su conjunto $(14,5 \%)$, sus «compadres» de las Administraciones eclesiásticas $(14,4 \%)$ y, por supuesto, que los más paupérrimos $(7,1 \%)$. El 22,2 \% que la nobleza rentista y dirigente del Concejo tenía inmovilizado en tales menesteres era, sin duda, el referente de los tesoreros generales. Todos ellos parvenus, llegados desde el comercio al por menor o al por mayor, según el caso, ansiosos por incorporarse a la cultura de las apariencias domésticas o del arreglo personal —en especial, en el vestuario y los adornos personales-. En ese sentido, si bien los porcentajes de la vestimenta de los tesoreros generales de Cruzada no eran muy elevados - Herrera, 4,3\%, Garma, 
4,8\%, y Tomé, 8,5\%-, el contraste con los promedios de la ciudad (11,9\%), con el de sus «compadres» $(9,6 \%)$, con el de los nobles rentistas $(8,6 \%)$ y con el de los menos afortunados en los niveles de patrimonio (20,8\%), deja entrever la importancia del atuendo en sus dinámicas existenciales aunque por debajo de aquellos y siempre acompañada la vestimenta con unos adornos personales muy significativos. Garma de la Puente sobresalía con diferencia. Las joyas y los complementos para el vestuario suponían el 22,7 \% de sus bienes muebles, prácticamente el doble que Herrera. Don Pedro Tomé no despuntaba en tal aspecto - con un escaso 6,4\%—. La tónica general en la ciudad, en los empleados en las Administraciones eclesiásticas y entre los nobles rentistas era del 11 al $16 \%$, con los más paupérrimos en montantes muy reducidos $(2,5 \%)^{41}$.

Con los menajes de cama ocurre algo similar a lo señalado para el vestido o incluso con el mobiliario ${ }^{42}$. Los porcentajes no son descollantes aunque sus cómputos nominales eran notables. Los tesoreros generales de la Santa Cruzada no pertenecían al pueblo pechero y más paupérrimo, que tenía inmovilizado en el vestuario $(20,8 \%)$, los menajes nocturnos $(16,9 \%)$ y el mobiliario doméstico $(15,2 \%)$ casi la mitad de sus bienes de entrecasa, con escasas pertenencias para la mesa, para el adorno personal y para el ornato de los interiores. Los bienes muebles de los más endebles socioeconómicamente suponían el 66,6\% de sus patrimonios, a gran distancia de los demás implicados en este análisis — sea la ciudad en su conjunto $(22,1 \%)$, la nobleza rentista $(24.4 \%)$ o los empleados en las administraciones eclesiásticas $(14,5 \%)$ - El perfil de los tesoreros les acerca a la aristocracia, en especial Garma $(7,1 \%)$ y Tomé $(6,8 \%)$, dotados de inmensas cantidades de dinero en efectivo ${ }^{43}$, deudas a su favor y substanciales bienes inmuebles. El devenir de los niveles de fortuna de don Pedro Tomé González ilustra, de manera paradigmática, a mi entender, tales asertos. A medida que fue incrementando sus volúmenes de negocio y de la asunción de asientos, decrecía en el porcentaje de lo inmovilizado en vestuario y en menajes de cama, e iba incrementando el índice, y los cómputos, de los menajes de mesa —-salvo excepciones-, de la decoración de sus interiores domésticos y de sus propiedades inmobiliarias libres — véase cuadro III—.

${ }^{41}$ Los 70 reales de dichos hogares contrasten nítidamente con los 12.000 reales de Herrera los 11.400 de Garma, los 6.400 de Tomé, los 4.000 de los empleados en las Administraciones eclesiásticas, los 5.600 de los nobles rentistas y, a la postre, los 1.600 reales de vellón del promedio de la ciudad en su conjunto (siglo XVIII).

42 Francisco SANZ de la Higuera, «Evolución de los lechos cotidianos y los menajes nocturnos en el XVIII burgalés», Cuadernos de Investigación Histórica (CIH), 28 (2011), págs. 389-431 y «Pajas, catres, cujas, camas, ...». El lecho cotidiano en el Antiguo Régimen: Burgos (1740-1780), Cuadernos de Investigación Histórica, 26 (2009), págs. 435-499.

43 Francisco Sanz de la Higuera, «Buena moneda y mala moneda en los hogares de Burgos en el siglo XVIII», Pecvnia, 18 (2014), págs. 13-40. 


\begin{tabular}{|c|c|c|c|c|c|c|c|c|c|}
\hline \multirow{2}{*}{ CUADRO III } & \multicolumn{9}{|c|}{ NIVELES DE FORTUNA DE PEDRO TOMÉ GONZÁLEZ } \\
\hline & \multicolumn{3}{|c|}{1703} & \multicolumn{3}{|c|}{1724} & \multicolumn{3}{|c|}{1765} \\
\hline ENSERES & Total (1) & $\%(2)$ & $\%(3)$ & TOTAL (1) & $\%(2)$ & $\%(3)$ & Total (1) & $\%(2)$ & $\%(3)$ \\
\hline Mobiliario & 554 & 6.2 & & 4.130 & 6.6 & & 8.307 & 10.8 & \\
\hline Vestuario & 2.904 & 32.8 & & 5.080 & 4.8 & & 8.655 & 8.6 & \\
\hline APEROS & & & & 967 & & & 8.213 & 1.1 & \\
\hline MENaJe DEL hOGar & 410 & 4.6 & & 1.763 & 13.4 & & 12.654 & 4.8 & \\
\hline Menaje de CAma & 990 & 11.2 & & 3.378 & 3.9 & & 6.170 & 7.6 & \\
\hline Menaje DE MESA & 1.544 & 17.4 & & 15.480 & 28.8 & & 26.450 & 20.2 & \\
\hline Menaje de Cocina & 272 & 3.1 & & 1.017 & 2.4 & & 2.321 & 2.1 & \\
\hline Objetos Religiosos & & & & 1.765 & 3.3 & & 3.184 & 4.9 & \\
\hline AdORNOS PERSONALES & 1.928 & 21.8 & & 4.508 & 22.7 & & 6.392 & 13.1 & \\
\hline Ocio/JuEGo & & & & 72 & & & 12 & 0.1 & \\
\hline Decoración & 248 & 2.8 & & 3.126 & 13.8 & & 11.417 & 22.2 & \\
\hline Libros & & & & & 0.3 & & & 0.9 & \\
\hline Transportes & & & & 500 & & & 8.573 & 3.6 & \\
\hline TOTAL BIENES MUEBLES & 8.850 & 100 & 20.6 & 41.786 & 100 & 12.3 & 102.080 & 100 & 6.8 \\
\hline BIENES INMUEBLES/RAÍZ & & & & 23.810 & & 7.1 & 192.657 & & 21.4 \\
\hline GANADO & & & & 1.936 & & 0.6 & 340.871 & & 1.5 \\
\hline RESERVAS ALIMENTOS & & & & & & & 26.827 & & 2.4 \\
\hline Tienda & 30.964 & & 72.2 & & & & 137.405 & & \\
\hline Dinero en EFECTIVO & 2.200 & & 5.1 & 156.868 & & 46.3 & 48.171 & & 12.2 \\
\hline Oficios & & & & & & & & & 7.2 \\
\hline DEUDAS A FAVOR & 867 & & 2.1 & 114.648 & & 33.8 & 661.465 & & 30.9 \\
\hline DEUDAS EN CONTRA & 10.788 & & 25.1 & 338.303 & & 99.8 & 186.144 & & 29.7 \\
\hline TOTAL PATRIMONIO & 42.881 & & 100 & 339.048 & & 100 & 1.509 .477 & & 100 \\
\hline
\end{tabular}

(1) Total: Promedio de cada ítem en reales de vellón.

(2) Porcentaje sobre el total de bienes muebles (enseres domésticos).

(3) Porcentaje sobre total del montante del inventario post-mortem (nivel de fortuna).

Fuente documental: Archivo Histórico Provincial de Burgos. Protocolos Notariales.

Véase Legajos en la nota 14.

La mención al dinero disponible en efectivo en el interior de sus casas materializa con contundencia el poderío económico de los tesoreros generales de la Cruzada. Don Juan Herrera acumulaba una cifra auténticamente impresionante, casi un cuarto de millón de reales de vellón, que significaban el 63,4 \% de su patrimonio y Garma de la Puente casi un tercio de millón $(42,4 \%)$. Tomé se descoloca en esa «foto» monetaria, tanto en volumen total de dinero como en el índice que supone sobre su nivel de fortuna $(3,2 \%)^{44}$. El contraste con la ciudad $(15,1 \%)$, sus «compadres» de las Administraciones eclesiásticas (11,1\%), los

${ }^{44}$ Compensado con el 43,8 \% de las deudas a su favor, que en Herrera eran de sólo el 3,9\%. 
nobles rentistas $(12,2 \%)$ y los hogares con menos de 10.000 reales $(5,7 \%)$ es categórico, en especial si comparamos los 236 1 줄 reales de estos últimos con las cifras indicadas para los tesoreros generales, preñadas sus estancias y sus talegos de oro y plata frente al ruin vellón de los pecheros ${ }^{45}$. Los tesoreros generales de la Santa Cruzada de la ciudad de Burgos estaban acostumbrados a manejar habitualmente mucho dinero en efectivo, sus hogares albergaban enormes masas monetarias de múltiples tipos de monedas y ello generaba las imprescindibles cautelas para su protección con las máximas garantías de seguridad ${ }^{46}$.

Desde una perspectiva más puntual y pormenorizada — véase cuadro IV— nos acercamos a algunos de los pertrechos más significativos de sus interiores domésticos, en especial a los muebles contenedores ${ }^{47}$, los menajes nocturnos ${ }^{48} \mathrm{y}$ los menajes para la disposición de la mesa ${ }^{49}$. Los tesoreros generales de la Santa Cruzada del Burgos del XVII y XVIII se caracterizaban por disponer de un volumen de enseres para cada uno de los pertrechos y menajes significados que les colocaba, en lo tocante a la disposición y disfrute de los interiores domésticos, entre la aristocracia más acaudalada y exuberante y los hogares menos afortunados de la ciudad. Se debatían, por tanto, entre quienes tenían mucho de casi todo y quienes se encontraban anclados en tener poco de casi nada ${ }^{50}$.

A los dichos tesoreros generales les gustaba rodearse de comodidad y confort, en especial en la orquestación de la mesa y el descanso nocturno, aunque sin excesivas exageraciones ni despilfarros. Se sabían unos parvenus, que escapaban a lo paupérrimo de los menos pudientes y a los boatos mayúsculos de

45 Francisco SAnZ de la Higuera, «Buena moneda y mala moneda...», Pecvnia, 18 (2014), págs. 13-40.

46 He desarrollado tal aserto, así como la hipótesis de que el incremento en la disponibilidad de dinero en efectivo en el seno de los hogares es signo inequívoco de recuperación económica, en Francisco SANZ DE LA Higuera, «Cultura material, dinero en efectivo y recuperación económica en el Burgos del XVIII», Stvdia Historica, Historia Moderna, (2014) (en proceso de revisión por el Consejo de Redacción).

47 Francisco Sanz de la Higuera, «Arcas, arcones, baúles y armarios. El mobiliario contenedor en el XVIII burgalés», en Francisco NúñEz RoLdán (Ed.), «Entre lo real y lo imaginario». Estudios de Historia Moderna en homenaje al profesor León Carlos Álvarez Santaló, Sevilla, Universidad de Sevilla y Huelva, 2014, págs. 461-478.

48 Francisco Sanz de la Higuera, «Evolución de los lechos cotidianos...», CIH, 28 (2011), págs. 389/411 y «Pajas, catres, cujas, camas,...», CIH, 26 (2009), págs. 435/499.

49 Francisco SAnZ de la Higuera, «Aproximación a la mesa de los burgaleses...», 2013, págs. 183-195 y «A la mesa de los burgaleses...», Investigaciones Históricas, 34 (2014), págs. 107-130.

50 Véanse a este respecto los análisis y propuestas de, por ejemplo, Belén Moreno Claverías, «Pautas de consumo y diferenciación social en El Penedés a fines del siglo XVII. Una propuesta metodológica a partir de inventarios sin valoraciones monetarias», en Enrique Llopis, Jaume Torras y Bartolomé Yun (eds.), El consumo en la España pre-industrial, Revista de Historia Económica, Número extraordinario, (2003), págs. 207245 (en especial: 225-240) y Consum i condicions de vida a la Catalunya Moderna. El Penedés, 1670-1790, Vilafranca del Penedés, Edicions i Propostes Culturals Andana, 2007 y, por supuesto, Fernand Braudel, Civilización material, economía y capitalismo. Siglos XV-XVIII. 1. Estructuras de lo cotidiano, Madrid, Alianza Editorial, 1984, págs. 233-252. 


\begin{tabular}{|c|c|c|c|c|c|c|c|c|c|c|}
\hline \multirow{3}{*}{$\begin{array}{l}\text { CUADRO IV } \\
\text { ENSERES }\end{array}$} & \multicolumn{10}{|c|}{ MENAJES CONTENEDORES, DE MESA Y DE CAMA } \\
\hline & \multicolumn{2}{|c|}{ HERRERA } & \multicolumn{2}{|c|}{ GARMA } & \multicolumn{2}{|c|}{ Tоме́ } & \multicolumn{2}{|c|}{ Burgos } & \multicolumn{2}{|c|}{ Admon Ecla } \\
\hline & $\mathrm{N}^{\mathrm{o}}$ & TASA (1) & $\mathrm{N}^{\mathrm{o}}$ & TASA (1) & $\mathrm{N}^{\mathrm{o}}$ & TASA (1) & $\mathrm{N}^{\mathrm{o}}$ & TASA (1) & $\mathrm{N}^{\mathrm{o}}$ & TASA (1) \\
\hline Cofres & 3 & 585 & 2 & 62 & 7 & 256 & 2 & 61 & 3 & 70 \\
\hline ArCONES & & & 1 & 2 & 9 & 566 & 1 & 63 & 2 & 125 \\
\hline ARCAS & & & 2 & 72 & 4 & 25 & 3 & 44 & 2 & 37 \\
\hline BAÚLES & & & 5 & 480 & 1 & 40 & 1 & 35 & 1 & 35 \\
\hline Armarios & 2 & 250 & 2 & 33 & 2 & 90 & 1 & 50 & 1 & 34 \\
\hline SÁBANAS & 24 & 648 & 25 & 705 & 62 & 1.776 & 18 & 446 & 20 & 567 \\
\hline Colchones & 8 & 275 & 23 & 456 & 15 & 830 & 8 & 368 & 10 & 432 \\
\hline MaNTAS & & & 11 & 124 & 7 & 270 & 7 & 119 & 7 & 130 \\
\hline Colchas & 1 & 1.000 & 6 & 316 & 15 & 1.093 & 6 & 266 & 7 & 322 \\
\hline Colgaduras & 3 & 7.780 & 1 & 220 & 2 & 1.450 & 2 & 838 & 1 & 388 \\
\hline CAMAS & 2 & 144 & 1 & 600 & 3 & 300 & 2 & 616 & 2 & 562 \\
\hline Catres & & & 4 & 48 & 3 & 110 & 2 & 105 & 1 & 33 \\
\hline Cujas & & & 2 & 16 & 2 & 20 & 3 & 27 & 3 & 24 \\
\hline TEnedores (PLATA) & 8 & 83 & 6 & 135 & 23 & 1.299 & 4 & 188 & 7 & 273 \\
\hline Cucharas (Plata) & 12 & 124 & 23 & 666 & 17 & 961 & 5 & 224 & 8 & 319 \\
\hline Cuchillos (Plata) & 12 & 60 & & & 6 & 320 & 1 & 50 & 1 & 38 \\
\hline Platos (Plata) & 2 & 524 & 15 & 3.945 & 12 & 1.375 & 1 & 317 & 2 & 392 \\
\hline \multirow[t]{2}{*}{ SERVILLETAS } & 36 & 120 & 65 & 136 & 117 & 542 & 25 & 91 & 32 & 103 \\
\hline & \multicolumn{2}{|c|}{ NoBLES } & \multicolumn{2}{|c|}{$<10.000 \mathrm{RV}$} & & & & & & \\
\hline ENSERES & $\mathrm{N}^{\mathrm{o}}$ & TASA (1) & $\mathrm{N}^{\mathrm{o}}$ & TASA (1) & & & & & & \\
\hline Cofres & 7 & 239 & 1 & 27 & & & & & & \\
\hline Arcones & 1 & 83 & 1 & 21 & & & & & & \\
\hline ArCAS & 4 & 72 & 2 & 30 & & & & & & \\
\hline BAÚLES & 3 & 88 & 1 & 11 & & & & & & \\
\hline Armarios & 2 & 157 & 1 & 17 & & & & & & \\
\hline SÁBANAS & 42 & 1.167 & 4 & 85 & & & & & & \\
\hline Colchones & 18 & 792 & 3 & 100 & & & & & & \\
\hline MANTAS & 12 & 222 & 4 & 60 & & & & & & \\
\hline Colchas & 13 & 1.167 & 2 & 79 & & & & & & \\
\hline Colgaduras & 2 & 1.843 & & & & & & & & \\
\hline Camas & 2 & 2.227 & 1 & 39 & & & & & & \\
\hline Catres & 3 & 129 & & & & & & & & \\
\hline Cujas & 4 & 33 & 2 & 13 & & & & & & \\
\hline TENEdORES (PLATA) & 13 & 659 & & & & & & & & \\
\hline Cucharas (PLATA) & 13 & 673 & 1 & 23 & & & & & & \\
\hline Cuchillos (Plata) & 5 & 227 & & & & & & & & \\
\hline Platos (Plata) & 7 & 2.176 & & & & & & & & \\
\hline SERVILLETAS & 80 & 334 & 7 & 19 & & & & & & \\
\hline
\end{tabular}

(1) Tasa: Promedio, en reales de vellón, de los enseres en los distintos hogares.

Fuentes documental: AHPB. PN. Múltiples Legajos. 
la noble rentista, titulada y dirigente del Concejo. Eran asentistas responsables de la distribución de un producto apetecible para la gran mayoría, o para toda, la población, las bulas, de vivos y de difuntos, de la Santa Cruzada, disponían de dinero en abundancia, de unos márgenes de beneficio significativos y de una posición en las redes de influencia de la monarquía. Estaban obligados a manifestar, hacia sus adentros domésticos y hacia sus afueras urbanos, callejeros, una cultura de las apariencias que les situaba entre los más importantes vecinos de la ciudad.

Y más allá —en la periferia de la muerte-

¿La cultura material y doméstica de los hogares de los tesoreros generales de la bula de la Santa Cruzada de Burgos en el XVII y XVIII se vio reflejada en sus disposiciones para después de su muerte ${ }^{51}$ A tenor de la documentación a que se ha tenido acceso, tras una ardua recopilación de archivo — véase cuadro $\mathrm{V}$-, Herrera $^{52}$, Garma ${ }^{53}$ y Tomé ${ }^{54}$ perfilaron en sus mandatos fúnebres y funerarios unas decisiones que reincidían en los parámetros que habían disfrutado durante su existencia terrenal. Se inclinaban más por ser inhumados en las parroquias urbanas que en conventos durante el Setecientos - Herrera designó el convento de San Pablo (1650)—. Garma de la Puente pidió ser revestido con el hábito de San Francisco de Asís, el atuendo funerario más común y habitual. Empero, Tomé González se decantó por el de Nuestra Señora del Carmen, bastante más exclusivista. En lo tocante a los acompañamientos durante los funerales y el

51 Véanse, entre otras muchas y excelentes publicaciones, las consideraciones y análisis de Máximo García Fernández, Los castellanos y la muerte..., Valladolid, Junta de Castilla y León, 1996. Antonio Rivas Álvarez, Miedo y piedad: testamentos sevillanos del siglo XVIII, Sevilla, Diputación Provincial de Sevilla, 1986 y Francisco Sanz de la Higuera, «Aristocracia eclesial en carrera de salvación: las dignidades catedralicias burgalesas a mediados del Setecientos», CIH, 24 (2007), págs. 355-393; «La terrible f(r)actura de la muerte...», CIH, 23 (2006), págs. 251/284 y «Vestiduras, hábitos, papeletas y ataúdes: el cadáver clerical en el Burgos del XVIII», Huerta de San Juan, Geografía e Historia, 12 (2005), págs. 215/246.

52 Las últimas voluntades de don Juan de Herrera, «estando enfermo de enfermedad natural», en AHPB. PN. Juan Díez del Real. Legajo 6514 (2 de agosto de 1650), folios 277-280.

53 El testamento de don Dionisio Garma de la Puente, «estando Como estoy Sano de Mi Cuerpo y en mi Juicio y enttendimientto nattural», en AHPB. PN. Lázaro Santamaría. Legajo 6862 (4 de enero de 1702), folios 23-26.

54 Para don Pedro Tomé González disponemos de dos testamentos. El primero, conjunto con su cónyuge, «no enfermos aunque Con algunos achaques havittuales y en nro sano Juicio y enttendimientto nattural», en AHPB. PN. Juan Antonio Fernández Guilarte. Legajo 7019 (16 de enero de 1754), folios 17-21), y el segundo, también en compañía de doña Josefa, e igualmente «no Enfermos aunque con algunos Achaques habittuales y en nro Sano Juicio y enttendimientto natural», custodiado en AHPB. Justicia Municipal. Juan Antonio Fernández Guilarte. Legajo 977 (16 de enero de 1765). 


\begin{tabular}{|c|c|c|c|c|}
\hline \multirow[t]{2}{*}{ CUADRO V } & \multicolumn{4}{|c|}{ MANDAS TESTAMENTARIAS Y DISPOSICIONES EN ÚLTIMAS VOLUNTADES } \\
\hline & HERRERA 1650 & GARMA 1702 & Tоме́ 1754 & Toмé 1765 \\
\hline НА́віто & & SAn Fco Asís & Ntra S Carmen & NTRA S Carmen \\
\hline INHUMACIÓN & Conv San Pablo & Parr SAn Lorenzo & Parr NS ViejarRúa & PARR NS VieJARRÚa \\
\hline Cruces ENTIERro & 4 & 5 & 3 & 3 \\
\hline ACOMPaÑAmiento & 2 & & 2 & 2 \\
\hline Niños Doctrina & SI & SI & SI & SI \\
\hline 12 PoBres & SI & & SI & SI \\
\hline $\mathrm{N}^{o}$ Misas ReZadas & 2.468 & 4.309 & 1.347 & 1.307 \\
\hline Coste Misas RV & 3.702 & 10.822 & 6.038 & 4.951 \\
\hline \multirow[t]{2}{*}{ Casa Sta Jerusalén RV } & & 24 & 100 & 100 \\
\hline & Burgos CIUdAD & Nobles Rentas & AdMON ECLA & $<10.000 \mathrm{RV}$ \\
\hline НА́віто & 55.8 & 72.5 & 84.2 & 67.6 \\
\hline NSC \% & 5.4 & 13.6 & 3.6 & 10.8 \\
\hline \multirow{2}{*}{ INHUMACIÓN } & 56.6 & 18.2 & 52.4 & 59.4 \\
\hline & 28.4 & 63.6 & 40.3 & 37.8 \\
\hline CRUCES ENTIERRO & 2 & 4 & 3 & 2 \\
\hline ACOMPAÑAMIENTO & 2 & 2 & 2 & 2 \\
\hline NiÑos Doctrina SI & 12.7 & 27.3 & 15.8 & 18.9 \\
\hline 12 Poвres SI & 10.3 & 13.6 & 12.2 & 10.8 \\
\hline $\mathrm{N}^{o}$ Misas Rezadas & 349 & 1.094 & 379 & 87 \\
\hline Coste Misas RV & 983 & 3.172 & 1.028 & 219 \\
\hline Casa Sta Jerusalén RV & 9 & 30 & 8 & 5 \\
\hline CAsa Jerusalén SI \% & 54.1 & 59.1 & 59.7 & 64.8 \\
\hline CONV= CONVENTO & PARR = PARROQUIA & & $\begin{array}{c}\text { RV }=\text { REALES DE } \\
\text { VELLÓN }\end{array}$ & \\
\hline SFA = SAN FCO Asís & NSC $=$ NTras $S^{\text {a Carmen }}$ & & & \\
\hline
\end{tabular}

Fuentes documentales: AHPB. PN. Múltiples Legajos.

tránsito del entierro solicitaron la presencia de parroquias y de hermandades y cofradías a la manera más aristocrática, la mayoría de ellos con los niños de la doctrina y 12 pobres a su vera. Además de con bula de difuntos y florín de San Juan, solicitaron ser enterrados con la papeleta correspondiente de la Casa Santa de Jerusalén, entidad a la que derivaron limosnas suculentas, en especial don Pedro Tomé, quien con 100 reales ocupó, junto con don Francisco Javier de Vega Río y Portocarreño ${ }^{55}$, el puesta más elevado de entre quienes efectuaban

55 Sus últimas voluntades en AHPB. PN. Francisco de Villafranca. Legajo 7099/4 (7 de febrero de 1773), folios 35-40. 
tales donativos a lo largo del siglo XVIII. En el número de misas rezadas y la asignación de desembolsos para tales menesteres, los tesoreros generales de la Santa Cruzada de Burgos demostraron estar a la altura de los aristócratas de la ciudad, solicitando ceremonias en los «Altares privilegiados» y «con la Mayor Brevedad ${ }^{56}$, con cómputos que reivindicaban su memoria tras el óbito en plena sintonía con las culturas materiales que les habían precedido en vida.

\section{A modo de conclusión}

Herrera, Garma de la Puente y Tomé González, tesoreros generales de la bula de la Santa Cruzada en el Burgos del Seiscientos y el Setecientos, no eran unos individuos pobres ni por sus venas corría una sangre excesivamente aristocrática. Asumieron una responsabilidad que comportaba notables fianzas, desembolsos y beneficios en lo relativo a la distribución de las bulas para vivos y las bulas para difuntos como telón de fondo. Aunque no eran poderosos banqueros ni rutilantes hombres de negocios, por sus manos y hogares transitaban inmensas cantidades de dinero. De tales manejos se derivaba su consolidación como asentistas de un producto que financiaba a la Monarquía hispánica en la medida de lo posible y les elevaba a una categoría notoria entre los vecinos de la ciudad, situados en la taxonomía socioeconómica y socioprofesional como avezados lugartenientes y testaferros de los más poderosos en La Corte. Sus esenciales quehaceres les afianzaban, sexenio tras sexenio, en una categoría de hogares muy selectos y exclusivos, dueños de unos niveles de renta y de fortuna, y de unas culturas materiales y de las apariencias acordes a sus importantes cometidos. Aunque parvenus, recién llegados al mundo de la riqueza y de la aristocracia urbana, gustaban de la buena mesa y de interiores domésticos, atuendos y adornos personales de significativa proyección, reflejados también sus gustos por el boato, la exuberancia y el confort en sus disposiciones fúnebres. Desde sus grandes camas de nobles maderas transitaban a sus descansos eternos en sepulturas de su propiedad, instaladas en conventos o en parroquias, donde se les recordaba por la largueza de sus mandas y la prodigalidad de sus desembolsos. Con la asunción de las responsabilidades de la distribución de la bula de la Santa Cruzada adquirieron un estatus muy elevado en el organigrama de las redes de influencia de una monarquía sedienta de ingresos y voraz en el despilfarro.

56 Tal y como se significa en el testamento de don Juan de Herrera en AHPB. PN. Juan Díaz del Real. Legajo 6515 (2 de agosto de 1650), folio $277 \mathrm{v}$. 